\title{
PERIODS RANGING FROM 5 TO 1500 DAYS \\ IN THE ANTICORRELATED MOVING LINES \\ OF SS 433*
}

\author{
S. FRASCA \\ Department of Physics, University of Rome \\ and \\ F. CIATTI and A. MAMMANO** \\ Asiago Astrophysical Observatory, University of Padova
}

(Received 6 October, 1983)

\begin{abstract}
Some tens of harmonic components are found in the moving lines of SS 433, leaving a standard deviation of 0.002 in $Z$ units, that is comparable to the observational error.

Ten harmonics appear anticorrelated in the two branches, among which those at 80,155 , and 1500 days are also strong. Their behaviour indicates changing structure in the ejecting mechanism.

No secular change is occurring in the precessional 163-day period beyond the estimated error of $5 \times 10^{-5} \mathrm{~d}^{-1}$. No periodicity occurs in beam velocity. One quarter of cases of $\left(Z_{1}+Z_{2}\right)$ are found outside their mean value by more than $5 \sigma$. This implies either variable beam velocity or absence of strict antiparallelism.
\end{abstract}

A harmonic analysis of 216 anticorrelated wavelengths of the relativistically displaced lines in SS 433 = V1343 Agl (Ciatti et al., 1983) has been performed, modelizing the results by a simulation program which avoids aliases due to spectral windows.

The red branch $\left(Z_{1}\right)$ can be represented with a standard deviation of 0.0026 (in $\Delta \lambda / \lambda=Z$ units) if 41 harmonic components, with periods ranging from 1 to 1500 days, are included. The blue branch $\left(Z_{2}\right)$ is still better represented by 63 harmonic components to \pm 0.0013 , i.e. at the observational error level (see Figures 1 and 3 ).

The most significant harmonics are listed in Table I. It appears that the phase differences of harmonics with about the same period in the two branches are nearly $180^{\circ}$, i.e. they result anticorrelated. A confirmation of their anticorrelation follows from harmonics found in the combination of $\left(Z_{1}-Z_{2}\right)$ data, which moreover supply nearly double amplitudes, while the $\left(Z_{1}+Z_{2}\right)$ data supply null results, as expected from anticorrelated harmonics.

Anticorrelated wavelength displacements are predicted in both disc and jet models for SS 433 (Cogotti et al., 1983), where periods of about 165, 6.28, 5.84, and 80 days agree with observations, given in Table I.

\footnotetext{
* Paper presented at the Lembang-Bamberg IAU Colloquium No. 80 on 'Double Stars: Physical Properties and Generic Relations', held at Bandung, Indonesia, 3-7 June, 1983.

** Also at Department of Mathematics, University of Messina.
} 


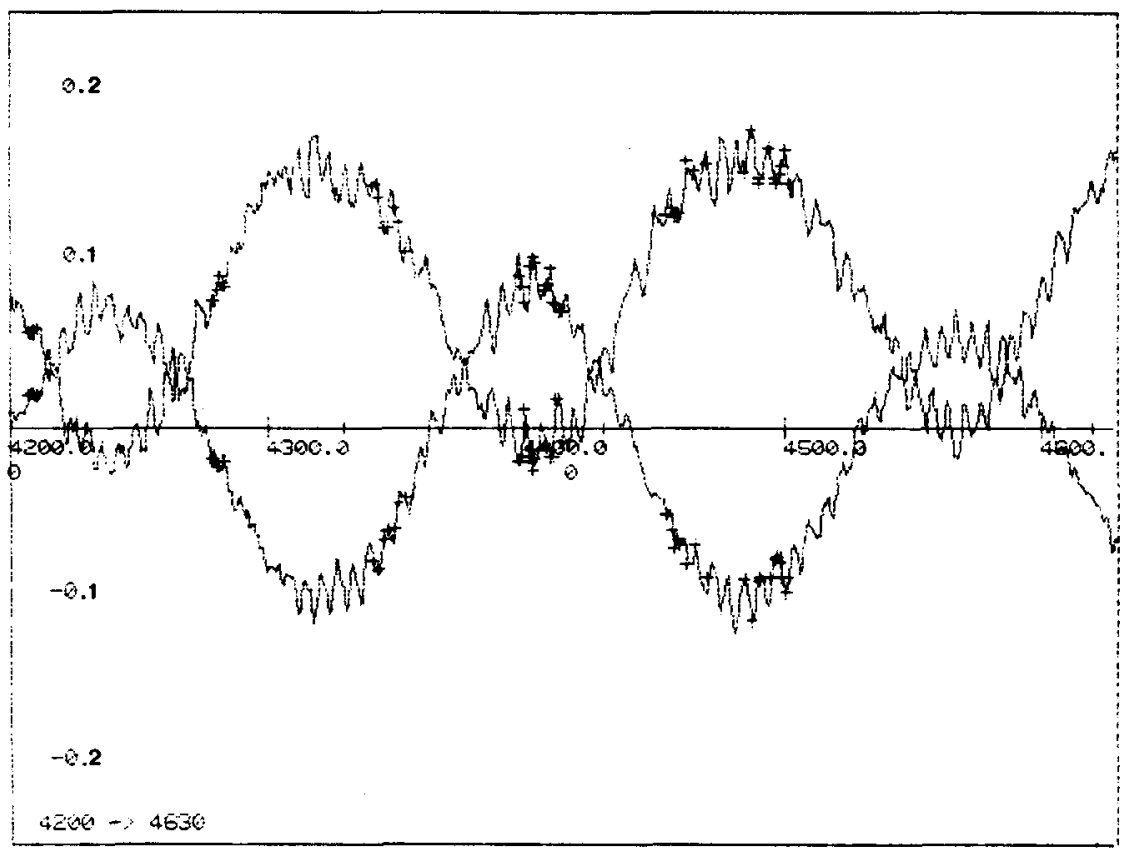

Fig. 1. Fit of SS 433 moving lines by means of 41 red and 63 blue harmonic components in the time interval $4200-4600 \mathrm{JD}$. Ordinates are in $Z$ units. Crosses represent observations.

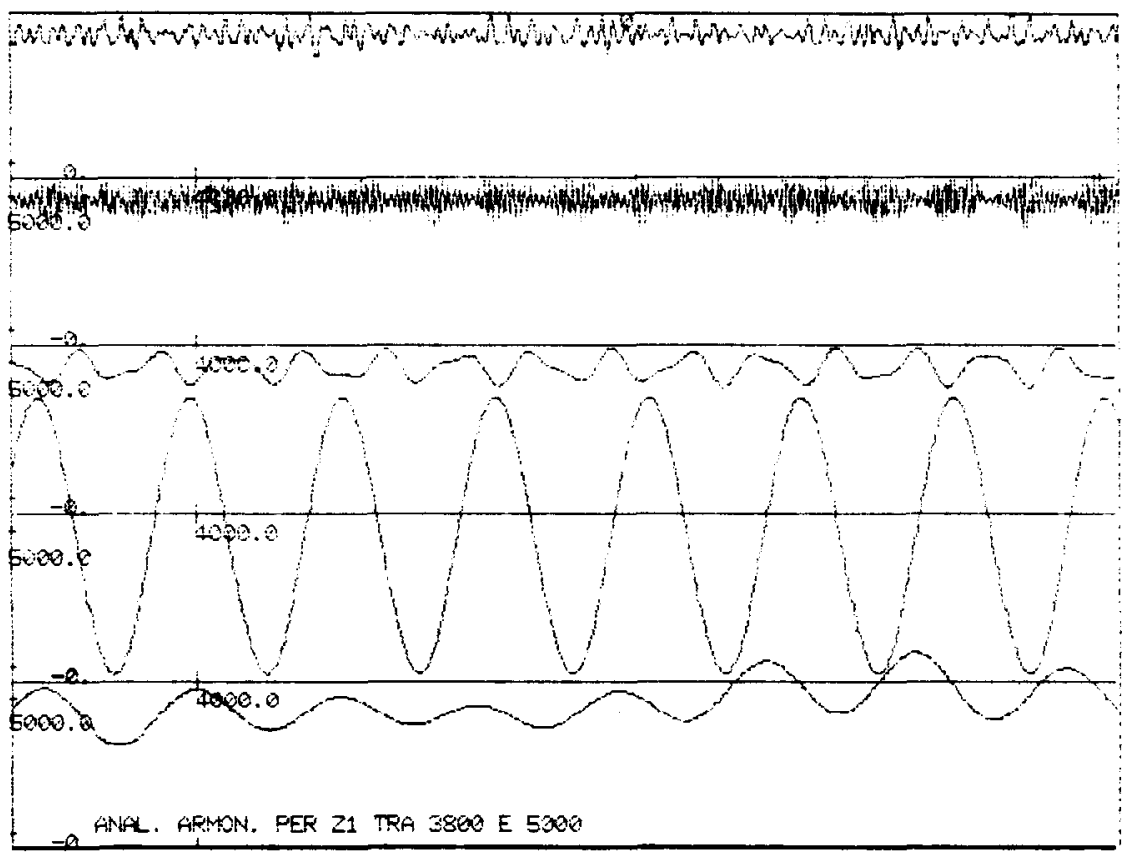

Fig. 2. Grouping of harmonic components of the red branch in SS 433 for all known observations from 3800 to $5000 \mathrm{JD}$. Ordinates are in $Z$ units. Note the variable behaviour of the 80 -day grouping and of the $150-180$ day grouping. 


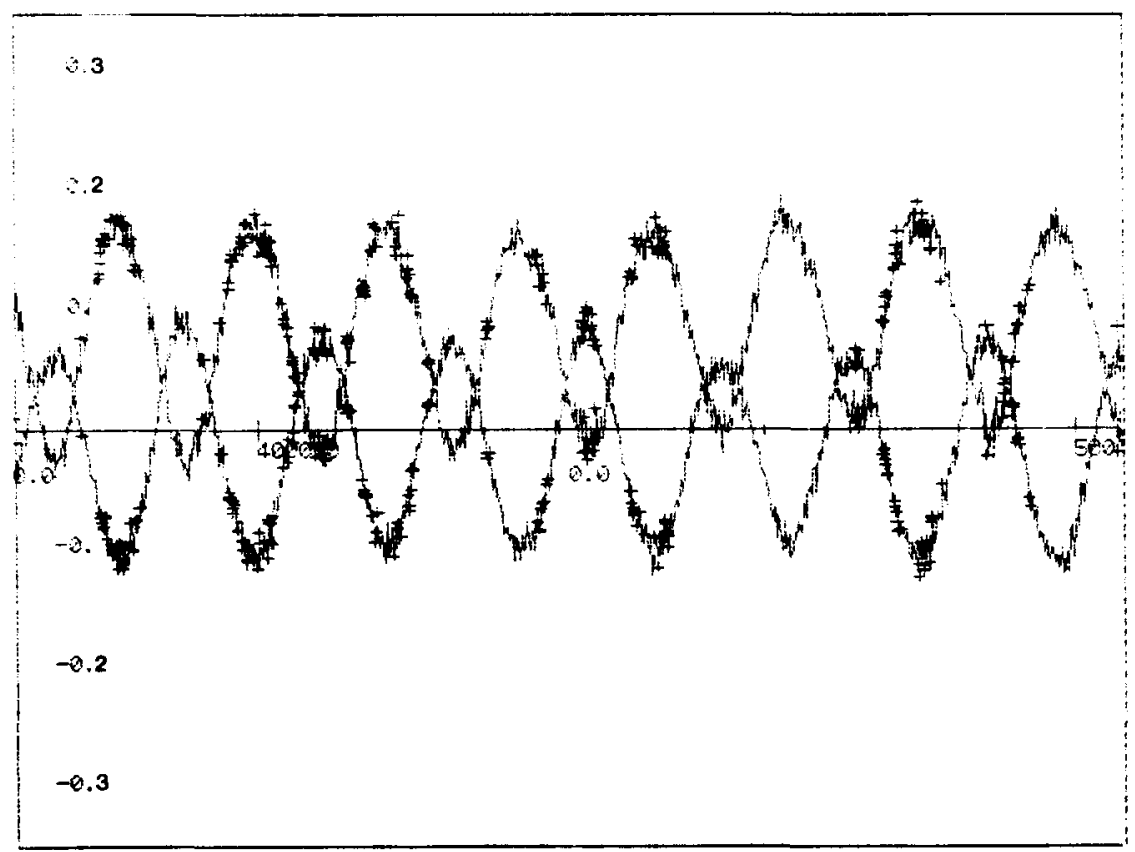

Fig. 3. Fit of the blue and red branches as in Figure 1, but in the time interval 3600-5000 JD, shows the strong influence of variable amplitude of some harmonic components. We can no more speak of a simple sinusoidal behaviour.

\section{TABLE I}

Most significant harmonic components in SS 433

\begin{tabular}{|c|c|c|c|c|c|}
\hline $\begin{array}{l}\psi \\
\text { period (amplitude) }\end{array}$ & \multicolumn{2}{|l|}{$\begin{array}{l}Z_{1} \\
\text { period (amplitude) }\end{array}$} & \multicolumn{3}{|c|}{$\begin{array}{l}Z_{2} \\
\text { period (amplitude) }\end{array}$} \\
\hline $164.8 \quad(18: 7)$ & $164.8 \pm 0.2$ & $(0.082)$ & 164.8 & $\pm \quad 0.2$ & $(0.083)$ \\
\hline $1311 \quad(2.7)$ & \pm 79 & $(0.009)$ & 1584 & \pm 121 & $(0.005)$ \\
\hline $153.8 \quad(2.0)$ & $156.6 \pm 0.9$ & $(0.011)$ & 155.7 & \pm 0.9 & $(0.011)$ \\
\hline $6.29 \quad(2.0)$ & $6.283 \pm 0.001$ & $(0.008)$ & 6.289 & $\pm \quad 0.001$ & $(0.008)$ \\
\hline $81.1 \quad(1.8)$ & $81.6 \pm 0.3$ & $(0.009)$ & & - & \\
\hline $77.9 \quad(1.3)$ & - & & 70.5 & \pm 0.3 & $(0.002)$ \\
\hline$(*)$ & $186.6 \pm 1.4$ & $(0.006)$ & 172.6 & \pm 1.2 & $(0.009)$ \\
\hline & & & 192.2 & \pm 1.6 & $(0.006)$ \\
\hline $5.84 \quad(1.1)$ & $5.833 \pm 0.002$ & $(0.005)$ & 5.839 & \pm 0.002 & $(0.004)$ \\
\hline $11.28 \quad(0.9)$ & - & & 11.28 & \pm 0.01 & $(0.003)$ \\
\hline $54.3 \quad(0.9)$ & $48.0 \pm 1.3$ & $(0.004)$ & & - & \\
\hline $6.54 \quad(0.9)$ & $6.793 \pm 0.004$ & $(0.002)$ & 6.541 & \pm 0.003 & $(0.002)$ \\
\hline $3.05 \quad(0.4)$ & - & & 3.086 & \pm 0.001 & $(0.002)$ \\
\hline- & $18.10 \pm 0.03$ & $(0.002)$ & 18.50 & \pm 0.03 & $(0.002)$ \\
\hline $4.21 \quad(0.4)$ & - & & 4.215 & \pm 0.001 & $(0.001)$ \\
\hline \multicolumn{6}{|l|}{ Standard deviation: } \\
\hline $0: 9$ & 0.0026 & & 0.0013 & & \\
\hline when using 26 periods & with 41 periods & & with $63 \mathrm{p}$ & periods & \\
\hline
\end{tabular}

* Periodicity of $182.5 \pm 1.3$ is present in $\left(Z_{1}-Z_{2}\right)$ data. 
We searched for periodicities in the direction of jets ( $\psi$, according to Ciatti et al., 1981) by assuming them antiparallel, and found 26 harmonic components whose periods again span from about 1 to 1300 days. The resulting standard deviation of 0.9 is close to the expected error inferred from the observations. Some of them are listed in Table I. We suggest that those harmonic components common at least to two of the parameters $Z_{1}$, $Z_{2}$, and $\psi$ are really anticorrelated and therefore may be among the most physically significant ones. Table $I$ lists just such harmonic components. The amplitudes in $Z_{1}$ and $Z_{2}$ are given in $\Delta \lambda / \lambda=Z$ units.

The standard deviations pertaining to the ephemeris, when using only the components in Table I, rise to 0.008 and 0.009 for the red and blue branch respectively, thus possibly indicating that some of the neglected components may also be significant.

We remark that the amplitudes of some components now discovered, namely those with periods about 80,155 , and 1500 days, are comparable to or larger than those of the nutation-like components at 6.28 and 5.84 days (Ciatti et al., 1983). Analysis of time subsets of the data show variable contributions of such individual long-period components versus time. One given period is being replaced in amplitude by one of the other two, as shown also in Figure 2, where the influence of groups of nearby components is represented. It thus seems that the structure of the body ejecting matter is slowly changing with time. In this connection we note that the time subsets show the components around 80 days to behave opposite to those of 165 days, thus excluding connections with its second harmonic.

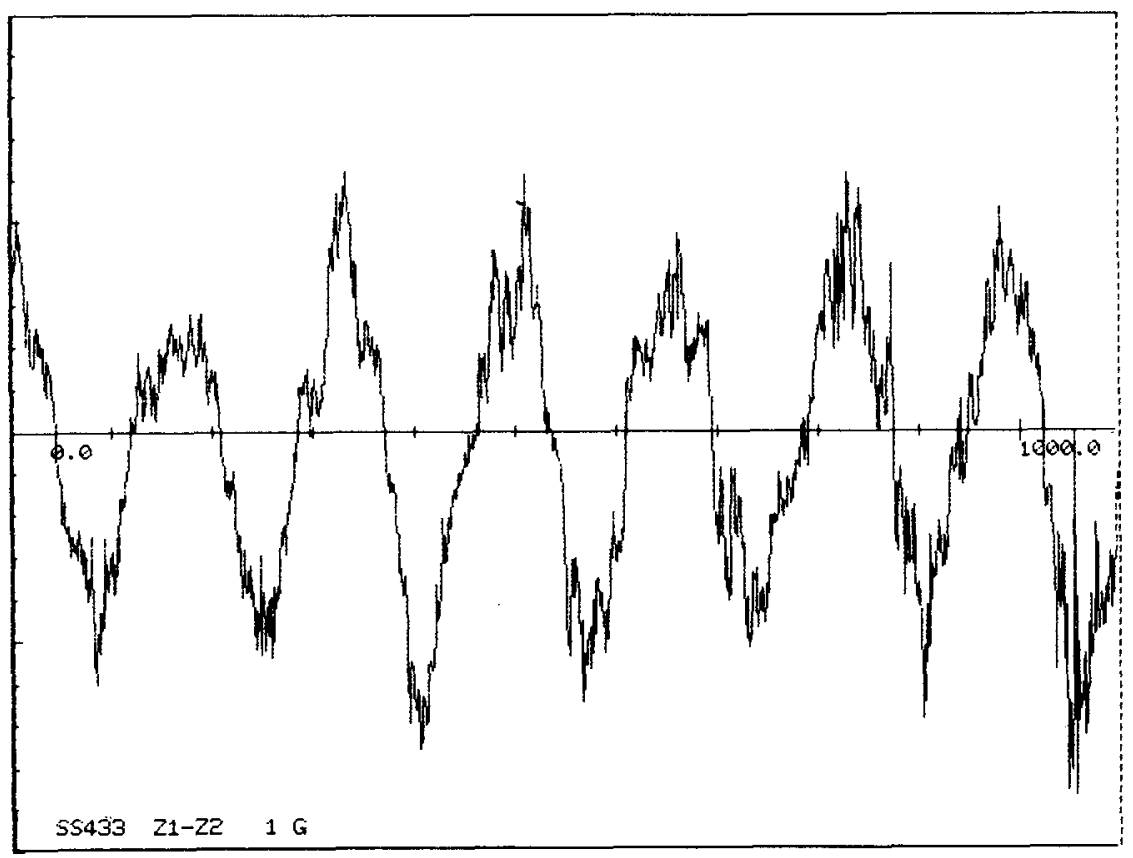

Fig. 4. Autocorrelation function of $\left(Z_{1}-Z_{2}\right)$ for 1000 days, with one day interval, showing no damping. 
However no secular trend is inferable from our data. While the 6.28-day component is stable in one part over $10^{4}$, analysis of the autocorrelation functions indicates that the precessional period has not changed beyond the estimated error of $0.8 \%$ (Figure 4).

Another important point is related to possible variability of beam velocity. Neglecting gravitational redshifts, the beam velocity would be a function of the combination $\left(Z_{1}+Z_{2}\right)$, if the jets were strictly antiparallel (Ciatti et al., 1981). We find in $25 \%$ of the cases the absolute values of the difference from the average are larger than 5 standard deviations. Therefore, if we assume constant beam velocity, the antiparallelism is not strictly true and viceversa.

Harmonic analysis in the antiparallelism hypothesis seems to show apparent periodicities in beam velocity from 5 to 238 days. However all their amplitudes are smaller than the standard deviation, so that their meaning remains doubtful. Moreover, by developing the formulae given by Milgrom (1980) in the assumption of a constant misalignment angle, the interferencies with the precessional period should appear in the red and blue branches, contrary to the observations. In this case we can therefore rule out periodicities in the beam velocity.

\section{Acknowledgements}

We thank Prof. M. Anile, A. Donato, G. Pizzella, L. Rosino, and R. Ruffini for useful discussions and for hospitality. This research has received financial support by the National Research Council, and by Ministry of Education under contract 3177 with University of Messina.

\section{References}

Ciatti, F., Mammano, A., and Vittone, A.: 1981, Astron. Astrophys. 94, 251.

Ciatti, F., Mammano, A., Iijima, T., and Vittone, A.: 1983, Astron. Astrophys. Suppl. Ser. 52, 443.

Cogotti, R., Doo Jong, S., and Ruffini, R.: 1983, preprint.

Milgrom, M.: 1980, Astron. Astrophys. 87, L15. 